BMJ

Open

Gastroenterology

\section{Outcomes of inflammatory bowel disease in patients with eosinophil- predominant colonic inflammation}

To cite: Alhmoud T, Gremida A, Colom Steele D, et al. Outcomes of inflammatory bowel disease in patients with eosinophil-predominant colonic inflammation. BMJ Open Gastro 2020;7:e000373. doi:10.1136/ bmjgast-2020-000373

Received 6 January 2020 Revised 22 January 2020 Accepted 27 January 2020

\section{Check for updates}

(C) Author(s) (or their employer(s)) 2020. Re-use permitted under CC BY-NC. No commercial re-use. See rights and permissions. Published by BMJ.

${ }^{1}$ Division of Gastroenterology and Hepatology, University of New Mexico Health Sciences Center, Albuquerque, New Mexico, USA

${ }^{2}$ Department of Internal Medicine, University of New Mexico Health Sciences Center, Albuquerque, New Mexico, USA ${ }^{3}$ Department of Pathology, University of New Mexico Health Sciences Center, Albuquerque, New Mexico, USA

Correspondence to Tarik Alhmoud;

talhmoud@gmail.com

\section{ABSTRACT}

Background Inflammatory bowel disease (IBD) is characterised by acute intestinal mucosal inflammation with chronic inflammatory features. Various degrees of mucosal eosinophilia are present along with the typical acute (neutrophil-predominant) inflammation. The effect of intestinal eosinophils on IBD outcomes remains unclear. Methods This is a retrospective study. Archived intestinal mucosal biopsy specimens of treatment-naïve IBD patients were examined by two pathologists. The number of eosinophils per high-power field was counted, and the mucosal inflammation was classified according to the eosinophilic inflammatory patterns. Clinical outcomes during the follow-up period were recorded.

Results 142 treatment-naïve IBD patients were included. Mean age was 39 years. $83 \%$ of patients had ulcerative colitis, and median follow-up was 3 years. $41 \%$ of patients had disease flare(s) and $24 \%$ required hospitalisation. Eosinophil count was not associated with risk of disease flare or hospitalisation. Patients with neutrophilpredominant inflammation ( $>70 \%$ neutrophils) had greater risk of disease flare(s): $27(55 \%)$ versus $24(36 \%)$ and $7(28 \%)$ in patients with mixed and eosinophil-predominant inflammation, respectively $(p=0.04)$. Overall, patients with neutrophil-predominant inflammation were more likely to have a disease flare; HR: $2.49,95 \% \mathrm{Cl}$ (1.0 to 5.6). Hospitalisation rate was higher in patients with neutrophilpredominant inflammation: $17(35 \%)$ compared to $17(19 \%)$ in patients with eosinophil-rich inflammation $(p=0.04)$. Kaplan-Meier analysis showed higher flare-free survival in patients with eosinophil-predominant inflammation compared to mixed and neutrophil-predominant inflammation.

Conclusion IBD patients with eosinophil-predominant inflammation phenotype might have reduced risk of disease flares and hospitalisation. Larger prospective studies to assess IBD outcomes in this subpopulation are warranted.

\section{INTRODUCTION}

Eosinophils are proinflammatory leucocytes, which normally reside in the gastrointestinal (GI) mucosa except the oesophagus. ${ }^{1}$ The quantity of eosinophils in different GI organs is variable. The colon has the highest number of eosinophils, where up to 50

\section{Summary box}

What is already known about this subject?

- Inflammatory bowel disease (IBD) patients may have increased mucosal eosinophils in the colon.

- The effect of eosinophils on IBD outcomes is unclear.

What are the new findings?

- Most IBD patients have increased mucosal eosinophils.

- Those with eosinophil-predominant inflammation prior to treatment had better outcomes.

How might it impact on clinical practice in the foreseeable future?

The degree of eosinophilic inflammation might help stratify IBD patients and predict disease outcomes.

eosinophils per high-power field (HPF) on light microscopy is considered normal. ${ }^{2-4}$ In the colon, eosinophils follow a quantitative gradient, being the highest in the right colon and lowest in the rectum. ${ }^{5}$ The number of intestinal mucosal eosinophils may rise in response to inflammatory conditions such as parasitic infections, coeliac disease, eosinophilic gastroenteritis and inflammatory bowel disease (IBD).$^{2-4}$ Patients with colitis such as IBD have increased mucosal eosinophils, even compared with patients who have food allergy. Eosinophils get activated in inflamed intestinal mucosa which leads to degranulation and release of eosinophilderived granule proteins such as major basic protein and eosinophil cationic protein (ECP) which can be cytotoxic. In addition, activated eosinophils secrete different cytokines, and can activate other immune cells such as mast cells. Activated eosinophils are thought to contribute to tissue inflammation and damage. ${ }^{3}$

Multiple immune cells are involved in IBD inflammation; mucosal histological examination in IBD patients typically shows active 
inflammatory cell infiltrates, consisting of neutrophils and neutrophil-filled crypts (crypt abscesses) associated with disease activity. Eosinophil-cell infiltrate can be present in IBD. In addition, chronic mononuclear infiltrate (mainly plasma cells) are present and can persist after remission of active disease. ${ }^{6}$

The role of eosinophils in different inflammatory diseases, particularly IBD, has become a point of major interest to clinicians and researchers. Wherever found in excess, eosinophils are thought to play a major proinflammatory role that is proportional to disease activity. ${ }^{7-9}$ The increased number of eosinophils is described in both Crohn's disease (CD) and ulcerative colitis (UC) patients; both in active disease and in disease remission. ${ }^{1011}$ Moreover, the number of activated eosinophils also correlates with disease activity. ${ }^{12} 13$ In murine model studies, dextran sulfate sodium was used to induce colitis in wild type and eosinophil-depleted animals. Using antibodies to ECP, the eosinophil-depleted animals were found to have less severe colitis, higher overall survival and less remodelling compared with wild type. ${ }^{1415}$

Furthermore, eosinophils may play a role in inflammation remodelling. A higher rate of disease recurrence after surgery in CD patients correlated with high-tissue eosinophil activity and overexpression of interleukin 5 (IL-5). ${ }^{16}$ Additionally, in a murine model, depletion of eosinophils using anti-CCR3 (eotaxin receptor) was found to be associated with less ileitis and remodelling. ${ }^{17}$ In UC patients, high eosinophilia was found to be linked to more severe colitis, higher need for surgery and higher incidence of primary sclerosing cholangitis compared with control UC patients without eosinophilia. ${ }^{18}$ While the exact mechanism by which eosinophils mediate inflammatory response in IBD has not been fully elucidated, some studies have suggested a potential profibrogenic role of eosinophils by enhancing fibroblast activity. ${ }^{19}$

Although many studies show proinflammatory role for eosinophils in IBD, others suggest that eosinophils may play a protective role. ${ }^{20} \mathrm{~A}$ study of UC patients reported that an increased number of activated eosinophils was associated with inactive disease. ${ }^{9}$ Eosinophils' anti-inflammatory role is thought to be due to production of protectin D1 (PD1). PD1 is the dominant antiinflammatory, proresolving mediator biosynthesized in human eosinophils. It negatively regulates eosinophils by suppressing their response to chemotaxins and expression of adhesion molecules such as CCR3. ${ }^{21} 22$

The significance of tissue eosinophilia in IBD remains unclear and controversial. The goal of this study is to describe the relationship of eosinophilic inflammation in IBD with the incidence of acute flares after diagnosis.

\section{METHODS}

This is a retrospective cohort study of IBD patients followed at the University of New Mexico between 1 January and 2000 and 30 November 2017; and have archived left-sided colon biopsies. The study was approved by the Institutional Review Board at the University of New Mexico. Health Insurance Portability and Accountability Act waiver of authorisation was obtained in accordance with guidelines and regulations of the Human Research Protection Office at the University of New Mexico. Cases with a clinical diagnosis of $\mathrm{CD}, \mathrm{UC}$ or indeterminate colitis; and involvement of the left colon were included. Cases were excluded if the initial diagnosis was not made at University of New Mexico, if there was not at least one follow-up in the IBD clinic, or if there was no tissue biopsy available prior to treatment. Right colon and ileal biopsies were not included in analysis due to small number of patients. Archived haematoxylin and eosin (H\&E) stained slides from cases of interest were reviewed by a fellowship-trained GI pathologist blinded to the clinical details of the cases. Cases with histologic changes inconsistent with IBD were excluded from the study, as were cases in which the original H\&E slides could not be retrieved.

Every slide of the left colon biopsies of each case was reviewed and evaluated for this study. Each slide was graded by disease severity into normal, mild, moderate and severe categories. The overall severity grade was determined by a composite overall evaluation of: amount of inflammation (acute and chronic), architectural distortion and the presence of ulceration/granulation tissue. Histologic severity was divided into mild, moderate and severe:

- Mild: increased inflammatory infiltrate in lamina propria with activity (active inflammatory cells involving crypt epithelium) involving a minority of crypts, with mild architectural distortion.

- Moderate: brisk inflammatory infiltrate in lamina propria with activity involving a majority of crypts, with at least mild architectural distortion.

- Severe: markedly injured and inflamed mucosa often accompanied with at least focal ulceration or granulation tissue. At least moderate architectural distortion.

For each patient, left colon biopsy slides with greatest inflammatory change were selected to evaluate eosinophilic inflammation and eosinophil count. The extent of neutrophilic and eosinophilic inflammation present in the epithelium and lamina propria of the mucosa was recorded for each slide involved by IBD. Each selected slide was categorised into eosinophilic predominant inflammation, neutrophilic predominant inflammation and mixed eosinophilic/neutrophilic inflammation. Majority of inflammation type determined ultimate characterisation:

- Neutrophil predominant: greater than $70 \%$ of active inflammatory cells (including lamina propria and crypts) comprised of neutrophils.

- Mixed: $30 \%-70 \%$ of active cells comprised of neutrophils or eosinophils.

- Eosinophilic predominant: greater than $70 \%$ of active inflammatory cells (including lamina propria and crypts) comprised of eosinophils. 
The presence or absence of eosinophilic crypt inflammation (eosinophilic cryptitis and crypt abscess) was recorded.

After these parameters were recorded by the senior pathologist, two pathology resident physicians performed formal quantitative eosinophil counts for each preselected slide. Each resident was trained to perform the counts in the same way. Each slide was screened at high magnification to identify the area containing the most eosinophil-rich inflammation (including lamina propria and epithelial eosinophils). The count was performed at 40x magnification, and only cells containing nuclei and coarse eosinophilic granules were included in the final counts. Cells containing granules disassociated from nuclei were excluded. If greater than 100 eosinophils were detected on the $40 \mathrm{x}$ field, the results were recorded as $>100$. For internal patient controls, eosinophil counts were performed on histologically normal biopsies in each biopsy series if available.

For all included cases, we conducted a chart review and extraction of relevant clinical and demographic data. Eosinophil count and classification of IBD inflammation at the time of diagnosis was performed. Predefined clinical outcomes were documented.

The primary outcome was the presence of one or more IBD flares requiring steroid therapy during the follow-up period. IBD flare is defined as a documented diagnosis of IBD flare by the treating gastroenterology physician during hospitalisation or office visit, and treatment with oral and/or intravenous steroids. Secondary outcomes included the need for hospitalisation, surgery (bowel resection); or development of abscess or fistula. The $\chi^{2}$ test was used to test if disease flares are related to different categories of eosinophilic inflammation. The Cochran Mantel-Haenszel statistics was used for further stratification by diagnosis (type of IBD) and disease severity.

The risk of the primary outcome during the follow-up period was calculated using Cox proportional HR; the model was adjusted for age, sex, race, type of disease and disease severity. Kaplan-Meier (KM) analysis with logrank testing to compare the proportion of patients in each category who remained free of a disease flare during follow-up was also performed.

Continuous variables were described in mean $\pm \mathrm{SD}$, we used median and IQR to describe continuous variables that are not normally distributed. The mean number of eosinophils in different disease categories were compared with a two-sided t test and $p$ value of 0.05 was considered significant. All statistical analysis was done using SAS V.9.4.

\section{RESULTS}

\section{Patient clinical characteristics}

Of note, 142 IBD patients met the inclusion criteria. Age was variable with a mean of $39 \pm 15.6$ years. Males were slightly higher in number and about two-thirds of patients were Caucasians. Follow-up was variable with a
Table 1 Patient characteristics and clinical outcomes

\begin{tabular}{|c|c|}
\hline Number of patients & 142 \\
\hline Age in years, mean $\pm S D$ & $39 \pm 15.6$ \\
\hline Sex, N (\%) & $82(58)$ \\
\hline Male & $82(58)$ \\
\hline \multicolumn{2}{|l|}{ Race, N (\%) } \\
\hline Caucasian & $89(63)$ \\
\hline African American & $7(5)$ \\
\hline Asian & $3(2)$ \\
\hline Native American & $2(1)$ \\
\hline Hispanic & $20(14)$ \\
\hline Others & 21(15) \\
\hline Length of follow-up in years, median \pm IQR & $3 \pm 5$ \\
\hline Number of IBD clinic visits, mean \pm SD & $11 \pm 11$ \\
\hline \multicolumn{2}{|l|}{ Diagnosis, N (\%) } \\
\hline Ulcerative colitis & $119(83)$ \\
\hline Pancolitis & $65(45)$ \\
\hline Left-sided colitis & $36(25)$ \\
\hline Ulcerative proctitis & $18(13)$ \\
\hline Crohn's colitis or ileocolitis & $19(13)$ \\
\hline Ileocolonic Crohn's & $9(6)$ \\
\hline Crohn's colitis & $10(7)$ \\
\hline Indeterminate colitis & $4(3)$ \\
\hline
\end{tabular}

Disease outcomes

Flares, N (\%)

$\begin{array}{ll}\text { No flares } & 83(59) \\ \text { At least one flare requiring corticosteroid } & 58(41) \\ \text { therapy } & \end{array}$
therapy

Hospitalisation, N (\%)

No hospitalisation 106 (76)

One or more hospitalisations for IBD flare or 34 (24) complication(s)

\begin{tabular}{ll} 
Surgery; bowel resection, N (\%) & \\
No need for surgery & $134(96)$ \\
One or more bowel resections & $6(4)$ \\
Fistula or abscess, N (\%) & $132(95)$ \\
No fistula & $7(5)$ \\
\hline
\end{tabular}

$\mathrm{IBD}$, inflammatory bowel disease.

median of 3 years and eight clinic visits. The majority of patients in this cohort $(83 \%)$ were diagnosed with UC. Most patients (59\%) had a benign course without flares during follow-up, while $41 \%$ of patients required at least one corticosteroid treatment. Two-thirds of those who underwent corticosteroid treatment $(65 \%)$ required hospitalisation. Only $4 \%$ of patients required bowel resection(s). Patient characteristics are summarised in table 1 . 

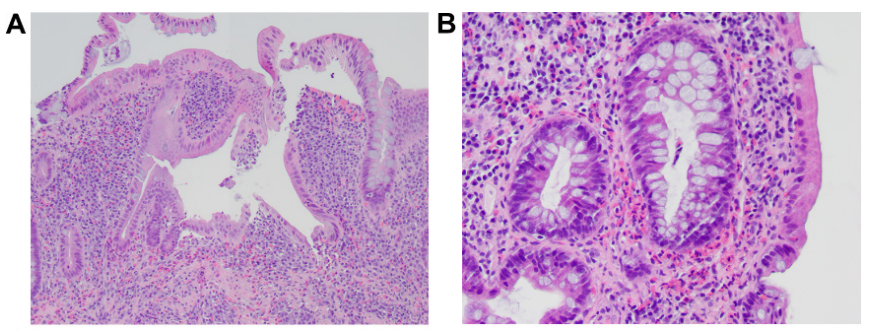

C
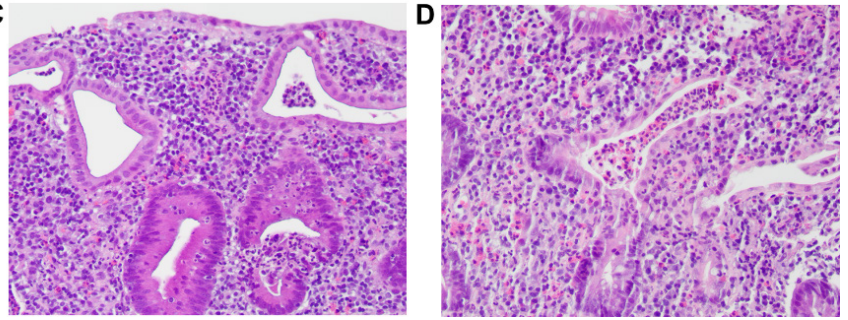

Figure 1 Hematoxylin and eosin stained slides of active inflammatory bowel disease showing different types of eosinophilic inflammation. (A) Chronic active colitis, eosinophil-predominant pattern. Colonic crypts show architectural irregularity and there is markedly increased lamina propria lymphoplasmacytic and eosinophilic inflammation. Neutrophils are inconspicuous. (B) Eosinophilpredominant pattern with enhanced lamina propria eosinophilic inflammation. (C) Chronic active colitis, neutrophil-predominant pattern. Distorted crypts with markedly increased lamina propria lymphoplasmacytic inflammation with scattered lamina propria neutrophils and eosinophils. Many intraepithelial neutrophils (active cryptitis) and neutrophilic microabscesses are present. (D) Chronic active colitis, mixed inflammatory pattern. Mixed eosinophilic and neutrophilic lamina propria inflammation with mixed eosinophilic/neutrophilic crypt abscesses (centre).

\section{Histological features of colon biopsies}

All specimens were reviewed by two pathologists and histological features of IBD were confirmed. Histopathologic severity of disease was moderate in nearly half of cases, while mild and severe inflammation were each noted in about one-quarter of patients (online supplementary table 1).

Eosinophil count per HPF is described by category: half of patients had a normal eosinophil count $(<50 / \mathrm{HPF})$, while about one-third had moderate count 50-100/ HPF and only $14 \%$ of patients had high counts greater than 100 eosinophils/HPF. Different types of inflammation were noted based on the abundance of eosinophils relative to neutrophils in the mucosal inflammatory infiltrate; a minority of patients $(17 \%)$ had eosinophilpredominant inflammation with inconspicuous neutrophils, meaning that most of the mucosal inflammatory cells were eosinophils (figure 1A,B); one-third of patients had neutrophil-predominant inflammation (figure 1C). However, half of patients had mixed eosinophil/neutrophil inflammation, where no cell type appeared to dominate in the examined histological sections (figure 1D).

Examination of the eosinophil inflammatory activity showed that only $23 \%$ of patients had evidence of eosinophilic cryptitis/abscess and $26 \%$ of all patients had severe colitis, which did not correlate with absolute eosinophil
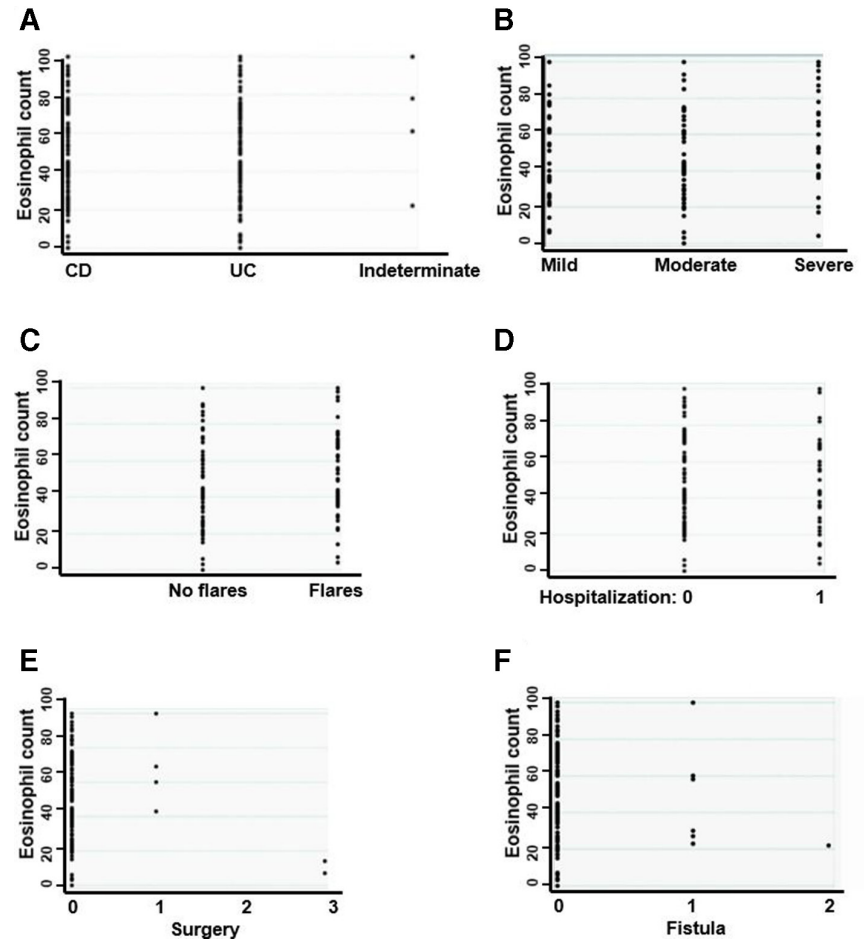

Figure 2 Mucosal eosinophil count in inflammatory bowel disease patients. (A, B) Eosinophil count by IBD subtype and disease histologic severity. (C, D) Eosinophil count by disease flare and need for hospitalisation ( 0 : no hospitalisation, 1: one or more hospitalisation(s)). (E, F) Eosinophil count by number of bowel resection surgeries or fistula formation (including perianal disease).

numbers. Characteristics of eosinophilic inflammation are described in online supplementary table 1.

\section{IBD type, histological severity and clinical outcomes based on eosinophil count}

Eosinophil count was not different between UC and CD (figure 2A), and was not associated with histological disease severity (figure 2B). There was no difference in eosinophil count between patients who had IBD flares versus those who remained in disease remission; mean eosinophil count \pm SD: $56.6 \pm 27$ versus $51.5 \pm 29.5$ $(\mathrm{p}=0.3)$, or in patients who were hospitalised compared with those who were not: $49.2 \pm 40$ versus $55 \pm 29$ ( $\mathrm{p}=0.3$ ) (figure 2C,D). Patients who had complicated CD with fistula formation or required bowel resection also had no difference in eosinophil counts (figure 2E,F). For more details on eosinophil count per outcome, please refer to online supplementary table 2 .

\section{Disease flares and other outcomes based on type of inflammation}

Patients with neutrophil predominant-inflammation at the time of diagnosis had greater risk of disease flare(s): $\mathrm{N}(\%) ; 27$ (55\%), compared with patients with mixed inflammation; $24(36 \%)$ and patients with eosinophil predominant inflammation; $7 \quad(28 \%) \quad(\mathrm{p}=0.04)$ (figure 3A). Patients with neutrophil-predominant inflammation had greater risk of disease flare(s) compared with 

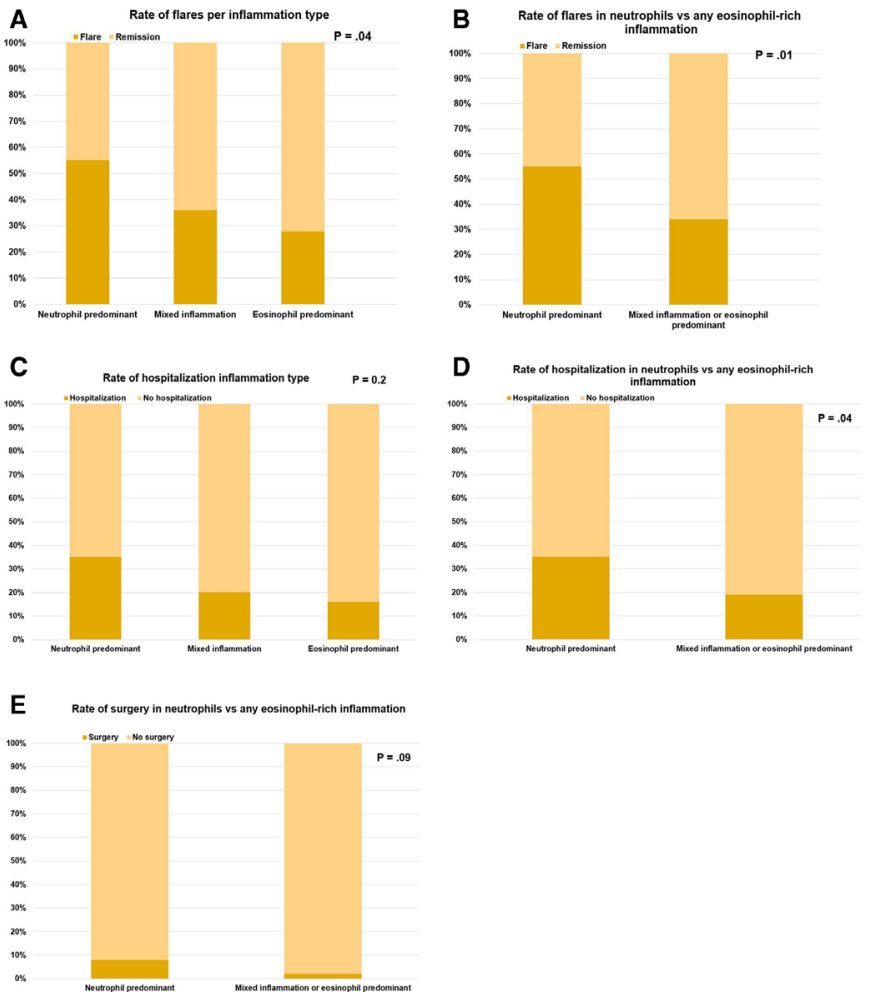

Figure 3 Outcomes per type of inflammation. (A) Rate of flare(s) per type of inflammation. (B). Rate of flare(s) in neutrophil-predominant versus eosinophil-rich inflammation (combined mixed-inflammation and eosinophil-predominant inflammation). (C, D) Rate of hospitalisation(s) per type of inflammation and in neutrophil-predominant versus eosinophil-rich inflammation. (E) Rate of surgery in neutrophil-predominant versus eosinophil-rich inflammation.

patients who had any type of eosinophil-rich inflammation (eosinophil-predominant and mixed inflammation groups combined) (figure 3B). After correction for disease severity and demographic variables; patients with neutrophil-predominant inflammation were more likely to have a disease flare HR: 2.49 , 95\% CI (1.0 to 5.6).

The risk of hospitalisation was higher in patients with neutrophil-predominant inflammation: 17 (35\%) compared with 17 (19\%) patients with eosinophil-rich inflammation $(\mathrm{p}=0.04)$. Increased risk of hospitalisation was not statistically significant when eosinophil-rich inflammation was stratified into mixed inflammation and
A Flare-free survival for all patients

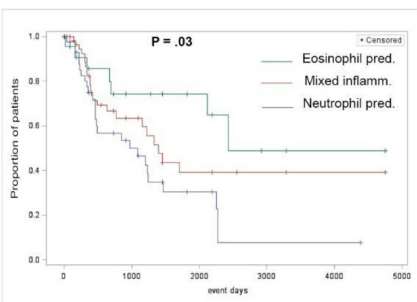

B Flare-free survival for patients with follow-up duration greater than three years

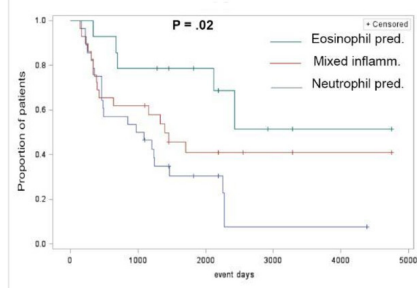

Figure 4 Kaplan-Meier analysis. (A) Proportion of patients who had disease flare(s). (B) Subgroup of patients who had follow-up more than 3 years. eosinophil-predominant inflammation (figure 3C,D) Other secondary outcomes including surgery (figure 3E) and fistula/abscess formation were not different based on type of inflammation. Online supplementary table 3 lists details of IBD type, severity and different outcomes per type of inflammation.

\section{Survival analysis based on type of inflammation}

$\mathrm{KM}$ analysis showed flare-free survival to be higher in patients with eosinophil-predominant inflammation over patients with mixed inflammation and neutrophilpredominant inflammation (figure 4A). KM analysis was similar among subjects who had long-term follow-up (greater than the study's median follow-up duration of 3 years) (figure $4 \mathrm{~B}$ ).

\section{Subgroup analysis of UC patients}

Data were analysed in the subgroup of patients who had UC. Rate of disease flares was higher in UC patients with neutrophil-predominant inflammation versus eosinophil-rich inflammation: 24 (56\%) versus 27 (36\%), $\mathrm{p}=0.04$. The result was not statistically significant when neutrophil-predominant inflammation was compared with mixed-inflammation and eosinophil predominant inflammation: $24(56 \%)$ versus $21(37 \%)$ versus $6(32 \%)$, $\mathrm{p}=0.1$. The rate of hospitalisation was higher in UC patients with neutrophil-predominant inflammation 16 (37\%) compared with patient with mixed-inflammation $9(16 \%)$ and eosinophil-predominant inflammation 3 (16\%), $\mathrm{p}=0.04$. Similarly, neutrophil-predominant UC patients had higher rate of disease flare compared with patients with eosinophil-rich inflammation 16 (37\%) versus $9(16 \%), p=0.01$. The results were not significant for $\mathrm{CD}$ patients probably due to the limited number of patients $(n=19)$. Outcomes in UC patients per type of inflammation are summarised in online supplementary table 4.

\section{DISCUSSION}

In our cohort, half of IBD patients with colitis had increased mucosal eosinophil count at the time of diagnosis prior to medical therapy, which is consistent with findings in a prior paediatric study. ${ }^{23}$ We examined all left colon biopsy specimens to categorise the inflammation as eosinophil-predominant, neutrophil-predominant and mixed inflammation, and found that IBD patients with neutrophil-predominant inflammation $(<30 \%$ eosinophils) had increased risk of a disease flare (HR: 2.49, 95\% CI (1.0 to 5.6) ) compared with patients with eosinophilpredominant inflammation $(>70 \%$ eosinophils). The percentage of disease flare was double in patients with neutrophil-predominant inflammation to eosinophilpredominant inflammation $55 \%$ versus $28 \%$, the risk was moderate for patients with mixed-inflammation (36\%), $\mathrm{p}=0.04$. The rate of hospitalisation was higher in patients with neutrophil-predominant inflammation (35\%) compared with patients with eosinophil-rich inflammation $(19 \%), p=0.04$. The absolute number of eosinophils 
did not predict risk of future flares or hospitalisation, rather the type of inflammation was more strongly associated with these outcomes.

Previous studies present controversial data about the role of eosinophils in IBD inflammation, and data regarding long-term outcomes based on the presence of eosinophil-rich inflammation are scarce. An early study with a limited number of patients $(n=50)$ suggested that ulcerative proctocolitis patients with a mild disease course had increased eosinophil counts compared with patients with more severe disease. ${ }^{20}$ A recent large paediatric study $(\mathrm{n}=368)$ showed that increased number of colonic eosinophils in treatment naive patient was associated with less severe inflammation on endoscopic examination. ${ }^{23}$ In contrast, other studies have shown that tissue eosinophils are associated with more severe disease. A study in paediatric patients $(\mathrm{n}=96)$ demonstrated that mucosal eosinophils greater than 60 per HPF / x 400 was correlated with corticosteroid therapy. ${ }^{24}$ In addition, other small studies have shown that severe eosinophilic infiltration is a significant predictor of poor response to treatment in UC patients. ${ }^{25} 26$

This study is unique because it focuses on the number of eosinophils and describes different types of eosinophilinflammatory phenotypes in IBD patients, and links these findings to disease outcomes. Other studies focused on the absolute number of eosinophils only and did not describe the global eosinophilic inflammation relative to neutrophils. This allowed us to classify the inflammation as neutrophil-predominant versus eosinophil-rich types (eosinophil predominant and mixed-inflammation).

There are a number of strengths of this study; a relatively large number of patients (142) were included. Importantly, all patients were treatment-naive at the time that biopsies were obtained. In addition, all specimens were read by an expert GI pathologist and follow-up information after diagnosis was available for all subjects, such that primary and secondary outcomes were confirmed by chart review.

This is a retrospective study and is liable to inherit limitations of the retrospective method. The primary outcome (disease flare) was based on chart review diagnosis, the number of disease flares can be underestimated. The treatment was not standard and performed by multiple gastroenterology physicians. We could not ensure that patients were compliant with medical treatment, however, it was verified that all patients were followed at the IBD clinic. The follow-up duration was variable; the median duration was 3 years and some patients only followed for few weeks, while other followed for more than 10 years. However, KM analysis was performed to account for the difference in follow-up times and the results were consistent between patients with short and extended follow-up durations. Additional studies need to explore the topic of eosinophilic-predominant inflammation in IBD patients.

IBD is a heterogeneous and multifactorial disease which causes significant morbidity for patients who fail to respond to medical treatment. Eosinophilic inflammation might play a role in explaining different IBD disease phenotypes, and variable response to different treatments and the variation in disease severity. Further studies are required to study the role of eosinophils in IBD disease outcomes.

Acknowledgements The authors thank Dr Roberta Redfern for her assistance in manuscript review.

Contributors TA: planned and conducted the study, data collection, analysis, manuscript writing. AG: data collection, data analysis and review, helped writing the manuscript. DCS, IF, WT, NN, MI, BAA: data collection. M-JX and AK: read pathology slides and reported eosinophil counts, helped with data analysis. DM: data collection, helped with study design and writing the manuscript, read pathology slides and described severity of colitis and type of inflammation.

Funding The authors have not declared a specific grant for this research from any funding agency in the public, commercial or not-for-profit sectors.

Competing interests None declared.

Patient consent for publication Not required.

Provenance and peer review Not commissioned; externally peer reviewed.

Data availability statement All data relevant to the study are included in the article or uploaded as supplementary information. All data are included in supplementary tables.

Open access This is an open access article distributed in accordance with the Creative Commons Attribution Non Commercial (CC BY-NC 4.0) license, which permits others to distribute, remix, adapt, build upon this work noncommercially, and license their derivative works on different terms, provided the original work is properly cited, appropriate credit is given, any changes made indicated, and the use is non-commercial. See: http://creativecommons.org/ licenses/by-nc/4.0/.

ORCID iD

Tarik Alhmoud http://orcid.org/0000-0002-1069-8532

\section{REFERENCES}

1 DeBrosse CW, Case JW, Putnam PE, et al. Quantity and distribution of eosinophils in the gastrointestinal tract of children. Pediatr Dev Pathol 2006;9:210-8.

2 Mehta P, Furuta GT. Eosinophils in gastrointestinal disorders: eosinophilic gastrointestinal diseases, celiac disease, inflammatory bowel diseases, and parasitic infections. Immunol Allergy Clin North Am 2015;35:413-37.

3 Woodruff SA, Masterson JC, Fillon S, et al. Role of eosinophils in inflammatory bowel and gastrointestinal diseases. J Pediatr Gastroenterol Nutr 2011;52:650-61.

4 Alhmoud T, Hanson JA, Parasher G. Eosinophilic gastroenteritis: an underdiagnosed condition. Dig Dis Sci 2016;61:2585-92.

5 Turner KO, Sinkre RA, Neumann WL, et al. Primary colonic eosinophilia and eosinophilic colitis in adults. Am J Surg Pathol 2017;41:225-33.

6 Bressenot A, Salleron J, Bastien C, et al. Comparing histological activity indexes in UC. Gut 2015;64:1412-8.

$7 \mathrm{Al}$-Haddad S, Riddell $\mathrm{RH}$. The role of eosinophils in inflammatory bowel disease. Gut 2005;54:1674-5.

8 Mishra A, Hogan SP, Lee JJ, et al. Fundamental signals that regulate eosinophil homing to the gastrointestinal tract. J Clin Invest 1999;103:1719-27

9 Lampinen M, Rönnblom A, Amin K, et al. Eosinophil granulocytes are activated during the remission phase of ulcerative colitis. Gut 2005;54:1714-20.

10 Wedemeyer J, Vosskuhl K. Role of gastrointestinal eosinophils in inflammatory bowel disease and intestinal tumours. Best Pract Res Clin Gastroenterol 2008;22:537-49.

11 Bischoff SC, Mayer J, Nguyen QT, et al. Immunnohistological assessment of intestinal eosinophil activation in patients with eosinophilic gastroenteritis and inflammatory bowel disease. Am J Gastroenterol 1999;94:3521-9.

12 Desreumaux P, Nutten S, Colombel JF. Activated eosinophils in inflammatory bowel disease: do they matter? Am J Gastroenterol 1999;94:3396-8. 
13 Smyth CM, Akasheh N, Woods S, et al. Activated eosinophils in association with enteric nerves in inflammatory bowel disease. PLoS One 2013;8:e64216.

14 Ahrens R, Waddell A, Seidu L, et al. Intestinal macrophage/epithelial cell-derived CCL11/eotaxin-1 mediates eosinophil recruitment and function in pediatric ulcerative colitis. J Immunol 2008;181:7390-9.

15 Shichijo K, Makiyama K, Wen C-Y, et al. Antibody to eosinophil cationic protein suppresses dextran sulfate sodium-induced colitis in rats. World J Gastroenterol 2005;11:4505-10.

16 Dubucquoi S, Janin A, Klein O, et al. Activated eosinophils and interleukin 5 expression in early recurrence of Crohn's disease. Gut 1995;37:242-6.

17 Masterson JC, McNamee EN, Jedlicka P, et al. Ccr3 blockade attenuates eosinophilic ileitis and associated remodeling. Am J Pathol 2011;179:2302-14.

18 Barrie A, Mourabet ME, Weyant K, et al. Recurrent blood eosinophilia in ulcerative colitis is associated with severe disease and primary sclerosing cholangitis. Dig Dis Sci 2013;58:222-8.

19 Xu X, Rivkind A, Pikarsky A, et al. Mast cells and eosinophils have a potential profibrogenic role in Crohn disease. Scand J Gastroenterol 2004;39:440-7.
20 Heatley RV, James PD. Eosinophils in the rectal mucosa. A simple method of predicting the outcome of ulcerative proctocolitis? Gut 1979;20:787-91.

21 Levy BD, Kohli P, Gotlinger K, et al. Protectin D1 is generated in asthma and dampens airway inflammation and hyperresponsiveness. J Immunol 2007;178:496-502.

22 Isobe Y, Kato T, Arita M. Emerging roles of eosinophils and eosinophil-derived lipid mediators in the resolution of inflammation. Front Immunol 2012;3:270.

23 Boyle B, Collins $\mathrm{MH}$, Wang Z, et al. Histologic correlates of clinical and endoscopic severity in children newly diagnosed with ulcerative colitis. Am J Surg Pathol 2017;41:1491-8.

24 Morgenstern S, Brook E, Rinawi F, et al. Tissue and peripheral eosinophilia as predictors for disease outcome in children with ulcerative colitis. Dig Liver Dis 2017;49:170-4.

25 Zezos P, Patsiaoura K, Nakos A, et al. Severe eosinophilic infiltration in colonic biopsies predicts patients with ulcerative colitis not responding to medical therapy. Colorectal Dis 2014;16:0420-30.

26 Leoncini G, Villanacci V, Marin MG, et al. Colonic hypereosinophilia in ulcerative colitis may help to predict the failure of steroid therapy. Tech Coloproctol 2018;22:941-6. 